\title{
Las finanza de petroleos mexicanos pemex II
}

\section{Petroleos mexicanos finances pemex II}

DOI: $10.46932 / \mathrm{sfjdv2n5-042}$

Received in: Jun 1st, 2021

Accepted in: Sep 30th, 2021

\author{
Héctor G. Riveros \\ Instituto de Física, UNAM \\ E-mail: riveros@fisica.unam.mx
}

\section{ERRORES EN LA CUENTA PÚBLICA Y LOS INFORMES MENSUALES DE PEMEX}

Pemex tiene un presupuesto predictivo, pero sus gastos reales están en la auditoria y la cuenta pública elaborada por Hacienda y aprobada por el Congreso.

Los datos de la Cuenta Pública 2018-2017-2016-2015, en billones de pesos son:

\begin{tabular}{|c|c|c|c|c|}
\hline \multicolumn{5}{|c|}{ Tabla I } \\
\hline Ventas de Bienes y Servicios & 1.681 & 1.397 & 1.079 & 1.166 \\
\hline Otros Ingresos y Beneficios & 2.886 & 5.887 & 11.361 & 5.255 \\
\hline Total, de ingresos y Beneficios & 4.567 & 7.285 & 12.441 & 6.421 \\
\hline Gastos de Funcionamiento & 0.999 & 0.821 & 0.768 & 0.740 \\
\hline Total Gastos y otras Pérdidas & 4.747 & 7,566 & 12.633 & 6.420 \\
\hline Pérdidas anuales & -0.180 & -0.281 & 0.191 & -0.712 \\
\hline
\end{tabular}

Los datos de las auditorias tienen las mismas pérdidas anuales, pero la Cuenta Pública [1] menciona ingresos por 5, 7, 12 y 6 billones de pesos. En gastos, mencionan cifras muy parecidas, de modo que las pérdidas sean iguales a las auditorias. El Presupuesto Federal para 2019 es de 5.8 billones de pesos. Sus principales ingresos debían ser de la venta de productos, pero reciben varias veces más de otros ingresos. Las petroleras dejan muy buenas ganancias, excepto en México. Los datos marcados no aparecen en las auditorias. En estos 4 años Pemex tuvo ingresos extras por 30.7 billones de pesos. Hacienda es responsable de esas cifras, aprobadas por el Congreso.

PEMEX: Las auditorias de Pemex [2], en cuatro años, de 2015 a 2018, NO MENCIONAN Otros ingresos, que son mencionados en la Cuenta Pública; dice que Pemex tuvo otros ingresos por 30.7 billones de pesos gastados sin incrementar sus activos de 2.075 billones o reducir sus pasivos de 3.535 billones de pesos. Pemex sigue en quiebra, no repara sus refinerías y compra gasolinas sin licitación y/o contaminadas. La nueva refinería, tendrá un costo de 0.16 billones de pesos. La cuenta pública dice que todo ese dinero se gastó, pero no menciona los detalles. Es claro que hay dinero para repartir con Hacienda, 
funcionarios de Pemex, auditores y el Congreso que aprobó los datos de Hacienda. Si recuperan estos ingresos, no pagaríamos impuestos por cinco años, con un presupuesto de 6 billones de pesos

En 2019 el total del presupuesto federal es de 5.8 billones de pesos, el 8\% del total se le asigna a Pemex como subsidio. Las compañías petroleras dejan ganancia, y pagan impuestos sobre sus ganancias. Hacienda debe explicar porque la cuenta pública de Pemex, menciona ingresos y gastos que las auditorias no conocen. Tienen que castigar a los autores de este Mega Fraude, muy superior a la Estafa Maestra. NO TIENE SENTIDO INVENTAR INGRESOS Y GASTOS, SIN OBTENER UN BENEFICIO ECONÓMICO. Con la complicidad de Pemex, de los auditores y el Congreso, quienes deben conocer estos datos, y no denunciaron el fraude. Video Pemex2019 https://youtu.be/_9n5UV2xjvI

Conocer un delito y no denunciarlo convierte en cómplices a muchos funcionarios, como investigador de la UNAM debo trabajar por mejorar a México. Este es una adición a un artículo publicado en Consultorio Fiscal de la UNAM, núm. 710, pág. 60-66 del 15 de marzo de 2019. https://www.dropbox.com/s/u4r3epjfynprz26/CF-

$710 \% 20 \mathrm{Pemex} \% 20 \mathrm{y} \% 20 \mathrm{su} \% 20$ situacion$\% 20$ financiera.pdf?dl=0

[1[ https://www.cuentapublica.hacienda.gob.mx/es/CP/Petroleos_Mexicanos-2017

[2] http://www.pemex.com/ri/finanzas/Resultados\%20anuales/dictamen.pdf

El gobierno dice que está reduciendo el robo de combustibles, y menciona que en noviembre de 2018 fue el mes de máximo robo, con 81 mil barriles diarios. Ver figura 1. La mejor ayuda para un gobierno es ayudarlo a mejorar. Veamos que nos dicen los informes de Pemex en Internet [3]. Comencemos por los datos de Petrolíferos en miles de barriles diarios Mbd.

Tabla II

$\begin{array}{cccccc}\begin{array}{c}\text { Petrolíferos } \\ \text { Mbd }\end{array} & \text { Ventas } & \text { Importación } & \text { Producción } & \text { Exportación } & \text { Pro+Imp-V-E } \\ \text { Octubre 18 } & 1407 & 990 & 598 & 116 & 65 \\ \text { Noviembre 18 } & 1419 & 1041 & 628 & 130 & 120 \\ \text { Diciembre 18 } & 1381 & 992 & 615 & 105 & 121 \\ \text { Enero 19 } & 1331 & 867 & 611 & 113 & 34 \\ \text { Febrero 19 } & 1404 & 968 & 716 & 104 & 176 \\ \text { Marzo 19 } & 1404 & 885 & 705 & 97 & 89 \\ \text { Abril 19 } & 1402 & 929 & 718 & 111 & 134 \\ \text { Mayo 19 } & 1392 & 833 & 754 & 117 & 78 \\ \text { Junio 19 } & 1379 & 869 & 786 & 103 & 173 \\ \text { Julio 19 } & 1406 & 769 & 786 & 100 & 49 \\ \text { Agosto 19 } & 1388 & 773 & 810 & 108 & 87 \\ \text { Septiembre 19 } & 1304 & 731 & 756 & 115 & 68\end{array}$


Tabla II.-Esta tabla menciona los datos de productos petrolíferos: vendidos, importados, producidos y exportados, en miles de barriles diarios, de octubre de 2018 a septiembre de 2019. La suma de Producción más Importación es mayor que el volumen de Ventas y Exportación, indicando la magnitud del robo. Todos los datos son mayores que $81 \mathrm{Mbd}$, y no muestran una tendencia a la baja.

En Productos Petrolíferos Pemex incluye diferentes productos según sea la tabla consultada. En Producción incluye gas licuado y otros, en Importación incluye gas licuado, gas natural, petroquímicos y crudo ligero, y en ventas internas incluye gas licuado, gas natural y asfaltos

Quizá el gobierno se refiere solamente a las gasolinas automotrices, Veamos los datos correspondientes.

\begin{tabular}{cccccc} 
& \multicolumn{4}{c}{ Tabla III } & \\
Gasolinas Mbd & Ventas & Importación & Producción & Exportación & Pro+Imp-V-E \\
Octubre 18 & 739 & 586.1 & 171.1 & 38 & -19.8 \\
Noviembre 18 & 737 & 608.2 & 177.7 & 26.2 & 22.7 \\
Diciembre 18 & 745 & 575.1 & 173 & 27.8 & -24.7 \\
Enero 19 & 708.8 & 513.6 & 153.1 & 35.1 & -77.2 \\
Febrero 19 & 730.2 & 591.1 & 217.1 & 30.2 & 47.8 \\
Marzo 19 & 722.9 & 542.5 & 208.8 & 34.5 & -6.1 \\
Abril 19 & 745.2 & 590.9 & 199.9 & 35.9 & 9.7 \\
Mayo 19 & 741.1 & 509.1 & 217.3 & 26.5 & -41.2 \\
Junio 19 & 723.8 & 555.5 & 215.1 & 39.5 & 7.3 \\
Julio 19 & 734.7 & 501.1 & 223.8 & 27.8 & -37.6 \\
Agosto 19 & 721.4 & 490.5 & 228.5 & 38.1 & -40.5 \\
Septiembre 19 & 688.1 & 430.7 & 203.1 & 26.4 & -80.7
\end{tabular}

Las ventas totales superan a la producción e importación. Solamente en noviembre 18 y en abril y junio19 hubo robo. Claramente las cuentas de Pemex no son compatibles entre sí.

Pemex sabe el robo diario. En los ductos miden la cantidad que entra al ducto y miden la que sale. En los depósitos, saben las cantidades de entrada y la cantidad en el depósito al iniciar actividades, saben las cantidades distribuidas y la cantidad en el depósito al acabar el día. Este último robo lo saben todos los días Y QUIEN ES EL RATERO, pero como tiene muchos cuates, no lo castigan.

Conociendo los volúmenes y entradas por la venta de gasolinas, se puede calcular los precios que Pemex cobra a las gasolineras y también el volumen y el precio que paga las gasolinas importadas, pero no dan precios separados para la Magna y la Premium importadas. Solamente conocemos el precio promedio en la siguiente Tabla IV [4]. 
Tabla IV

\begin{tabular}{|c|c|c|c|}
\hline Gasolinas Mbd & Magna & Premium & Importada \\
\hline Enero 19 & 9.11 & 10.29 & 7.94 \\
\hline Febrero 19 & 9.77 & 10.40 & 7.62 \\
\hline Marzo 19 & 10.76 & 11.63 & 9.69 \\
\hline Abril 19 & 11.71 & 12.45 & 10.37 \\
\hline Mayo 19 & 11.73 & 12.32 & 11.22 \\
\hline Junio 19 & 10.61 & 11.87 & 10.17 \\
\hline Julio 19 & 11.16 & 12.11 & 10.30 \\
\hline Agosto 19 & 10.42 & 11.78 & 9.97 \\
\hline Septiembre 19 & 10.55 & 11.75 & 9.03 \\
\hline
\end{tabular}

Precios calculados en pesos/litro de gasolina. Para Magna y Premium eso les cobra a las gasolineras, que nos cobran los impuestos y su ganancia. Antes era de menos de un peso por litro y la fijaba Hacienda. Los precios de la gasolina importada son los pesos que paga entre los litros importados, que no dice cuántos litros son de cada tipo.

Pemex prefiere importar gasolina que le permite ganar más, su negocio es revender. Por eso las refinerías están abandonadas. Le quieren dar 100 mil millones de pesos de nuestros impuestos, cuando sus pérdidas anuales son mucho mayores que esa cantidad. Lo peor es que las Cuentas Públicas de 2015 a 2018 mencionan ingresos por 30 billones de pesos que se gastaron sin disminuir sus deudas y sin incrementar sus inversiones. Pemex no es de México, México es de Pemex. No se ve fondo al pozo que constituye Pemex y el presidente ni siquiera ve el pozo. Como extraño a Juárez. Juárez y su excelente gabinete sacaron a México del caos.

El gobierno publica datos optimistas de su campaña contra el huachicol, como llaman al robo de combustibles. No menciona el origen de sus datos, los obreros de Pemex saben exactamente cuanto se pierde en los ductos y cuanto se roban de los depósitos mediante el uso de pipas de procedencia desconocida para nosotros, pero son sus conocidos o parientes. Es preocupante que se perforen los tubos, por el peligro latente de incendios, además del robo es necesario curar a los ladrones con los gastos correspondientes. Entiendo que se les cure por razones humanitarias, pero no entiendo que no se les aplique la Ley. Aunque este comportamiento del gobierno es compatible con premiar con plazas automáticas a los normalistas que secuestran centenas de autobuses con todo y sus choferes. Lo más grave es que les confiaremos la educación de los niños mexicanos más desvalidos, enseñándoles que la violencia es la solución de todos los problemas. 
Fig.1.-Esta gráfica muestra la versión oficial y el video muestra la versión no oficial Pemex2019 https://youtu.be/_9n5UV2xjvI

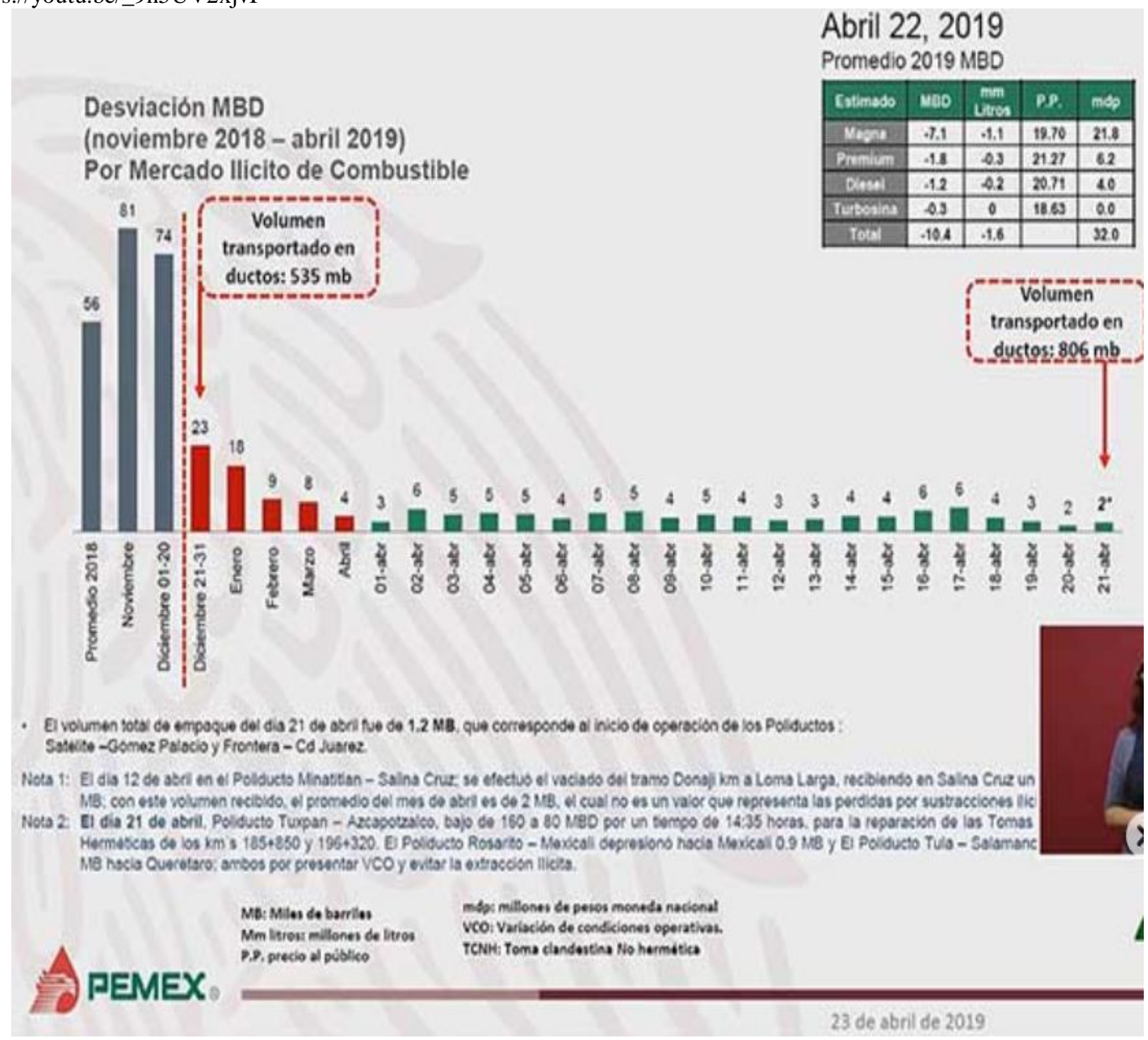

Pero el gobierno inventa cifras sin respaldo. Pemex ahora dice que roban lo mismo que antes. No se les puede creer.

\section{BALANZA COMERCIAL DE PEMEX.}

Se nos dijo que el gasolinazo se debió a que el precio internacional de las gasolinas subió, pero los precios de la gasolina en estados Unidos son bastante estables y más barata que en México. El 100\% de las ganancias del gasolinazo pasó a Hacienda, las gasolineras ganan de 91 a 92 centavos por litro de gasolina vendido. Muchos años creí que Pemex no crecía porque el gobierno se quedaba con las ganancias que producía Pemex. Revisando el presupuesto 2017 me sorprendió encontrar que Pemex recibió 492 mil millones de pesos, de los impuestos que pagamos todos los mexicanos. En 2014 se le asignaron 540, y en 2015 fueron 478 mil millones de pesos. Se está subsidiando a una empresa quebrada por la codicia de ella 
misma. Le está regalando dinero de nuestros impuestos a una empresa que debería generar ganancias y pagar impuestos. Pemex necesita quebrar y refundarla como compañía sustentable. Desde 2015, las exportaciones de Pemex son inferiores a sus importaciones, se compra gasolina porque desperdicia su capacidad instalada. Para que hacerles otra refinería si no saben usar las que ya tienen. En el mundo, hay refinerías cerradas por falta de mercado, deben estar en barata.

Como Universitario me enorgullece estudiar problemas nacionales y cumplir con la Ley Orgánica. Hay quienes no entienden que la Universidad debe estar al servicio de México, México nos necesita. Felicito a Consultorio Fiscal en ayudar al progreso de México. 


\section{REFERENCIAS}

Cuenta Publica https://www.cuentapublica.hacienda.gob.mx/es/CP/Petroleos_Mexicanos-2017

Auditorias http://www.pemex.com/ri/finanzas/Resultados\%20anuales/dictamen.pdf

Datos mensuales Estadisticas Petroleras.

https://www.pemex.com/ri/Publicaciones/Indicadores\%20Petroleros/indicador.pdf

Valor importaciones Gasolina Pemex Dirección Corporativa de Planeación, Coordinación y Desempeño. Apéndices A y B 


\section{APENDICE A}

\begin{tabular}{|c|c|c|c|c|c|c|c|c|c|}
\hline Petróleos Mexicanos & & & & & & & & & \\
\hline \multicolumn{10}{|c|}{ Dirección Corporativa de Planeación, Cacrdinación y Desempeño } \\
\hline \multicolumn{10}{|c|}{ Valor del comercio exterior de productos petroliferos y gas natural } \\
\hline \multicolumn{10}{|c|}{ (millanes de dobloses) } \\
\hline & & & & & & & & & \multirow[b]{3}{*}{ Sep/2019 } \\
\hline & \multicolumn{2}{|l|}{ OBSERVAT } & \multirow[b]{2}{*}{ Mar/2019 } & \multirow[b]{2}{*}{ Abr/2019 } & \multirow[b]{2}{*}{ May/2019 } & \multirow[b]{2}{*}{ Jun/2019 } & \multirow[b]{2}{*}{ Jul/2019 } & \multirow[b]{2}{*}{ Ago/2019 } & \\
\hline & Ene/2019 F & Feb/2019 & & & & & & & \\
\hline Exportaciones netas & -1689.84 & -1781.58 & -2042.17 & -2195.37 & -2093.7 & -2012.22 & -1794.6 & -1753.85 & -1470.58 \\
\hline Exportaciones & 171.7587 & 161.7965 & 170.4691 & 191.8154 & 204.5017 & 152.8658 & 157.0891 & 151.5316 & 165.3644 \\
\hline \multirow[t]{2}{*}{ Petroliferos } & 171.6567 & 161.7328 & 170.4004 & 191.7505 & 204.4444 & 152.8073 & 157.0455 & 151.4908 & 165.3194 \\
\hline & & & & & & & & & \\
\hline Gas licuado (1) & NDD & NID & ND & NDD & NID & ND & NDD & NID & ND \\
\hline Pentancs (1) & ND $\quad A$ & NID & N:D & ND & NID & NID & NDD & NID & ND \\
\hline Gasolinas (1) & \multicolumn{2}{|c|}{49.286057541 .2495200} & 56.4500473 & 39.8076372 & 44.4871985 & 54.2647401 & $\frac{141,069627 \%}{\mathrm{NDP}}$ & 51.012725 & 36.7333342 \\
\hline Turbosina & NDD $\quad A$ & NiD & NID & ND & N/D & N/D & ND & NID & ND \\
\hline Diesel & NDD & NID & NID & NID & NID & ND & NDD & NID & NID \\
\hline Gasobleo de vacio & NDD $\quad A$ & NiD & N/D & ND & N/D & ND & ND & N/D & NID \\
\hline Combustóleo & \multicolumn{2}{|c|}{$118,131864118,18235$} & 96.3697608 & 113.056398 & $117.87746 E$ & 69.878268 & 107.72522: & 50.3106965 & 595.0735771 \\
\hline Asfaltos & NID & NID & ND & NDD & NID & ND & NDD & NID & NID \\
\hline Residuo largo & ND $\quad$ A & NID & NID & ND & N/D & ND & ND & NID & \multirow{2}{*}{$\begin{array}{l}\text { NID } \\
9133.5124514\end{array}$} \\
\hline Otros (2) & \multicolumn{2}{|c|}{$4.2387475: 2.30090915$} & $\$ 17.5806197$ & 718.8864968 & 5.42 .0797016 & 28.6642825 & 68.25066847 & 20.1673291 & \\
\hline Gas natural (1) & 0.102004 & 0.063713 & 0.068645 & 0.064859 & 0.057336 & 0.058498 & 0.043581 & 0.040838 & 0.045022 \\
\hline Gas naturain (i) & & & & & & & & & \\
\hline Importaciones & 1861.6 & 1943.38 & 2212.638 & 2387.187 & 2298.201 & 2165.082 & 1951.688 & 1905.382 & 1635.941 \\
\hline \multirow[t]{2}{*}{ Petroliferos } & 1727.172 & 1841.181 & 2104.079 & 2294.236 & 2201.853 & 2072.987 & 1870.753 & 1832.699 & 1559.45 \\
\hline & & & & & & & & & \\
\hline Gas ficuado (1) & $21.004668 c 1$ & 17.2005242 & 12.9919536 & E 11.1246982 & 212.3904068 & 9.70914835 & 5. 9.5251217 & 9.82068227 & 10.0651097 \\
\hline Propano (1) & 55.36400956 & 65.3960405 & 44.7901398 & 47.9001795 & $\leqslant 32.555613 t$ & $35.359008 \mathrm{e}$ & 20.0114531 & 16.6211126 & 37.8963271 \\
\hline Gasolinas & 1005.947861 & $1109.8526 \subseteq$ & 1295.2592 & 1510.20608 & $\$ 1407.30498$ & 1391.02601 & 11271.5596 & $1203.8525 \mathrm{t}$ & 958.575647 \\
\hline Naftas & 46.52828713 & 35.5322142 & 40.687928 & 20.8049711 & $518.917540:$ & 18.2275327 & 39.4940273 & $538.530262:$ & 37.0255202 \\
\hline Diesel & 441.239135 4 & $466.87093 \mathrm{E}$ & 516.518535 & 526.75162 & 519.808811 & 457.402602 & 2357.148197 & 7390.467664 & 377.240645 \\
\hline Combustóleo & 7.187264267 & 7.67275176 & 33.2516756 & 17.1615208 & 559.8284605 & 44.8982181 & 117.9658395 & $527.686035 t$ & 20.2321333 \\
\hline Otros & 149.9005641 & 138.65633 & 160.579138 & 160.28673 & E 151.047585 & 116.364681 & 155.058597 & 7 145.740691 & 118.414209 \\
\hline & & & & & & & & & \\
\hline Gas natural (1) & 134.4283 & 102.1988 & 108.5595 & 92.95109 & 96.34744 & 92.09516 & 80.93529 & 72.68263 & 76.49101 \\
\hline (1) Transaociones realizadas p & quimica Basicic & ca. En gasoli & linas, & & & & & & \\
\hline induye las gasolinas noturales & sta subsidaria & & & & & & & & \\
\hline (2) Incluye condensados. & & & & & & & & & \\
\hline
\end{tabular}


